\title{
BMJ Open Non-psychotropic medication and risk of suicide or attempted suicide: a systematic review
}

\author{
Hayley C Gorton, ${ }^{1,2}$ Roger T Webb, ${ }^{3}$ Navneet Kapur, ${ }^{3}$ Darren M Ashcroft ${ }^{1,2}$
}

To cite: Gorton HC, Webb RT, Kapur N, et al. Non-psychotropic medication and risk of suicide or attempted suicide: a systematic review. BMJ Open 2016;6:e009074. doi:10.1136/bmjopen-2015009074

- Prepublication history and additional material is available. To view please visit the journal (http://dx.doi.org/ 10.1136/bmjopen-2015009074).

Received 12 June 2015 Revised 24 August 2015 Accepted 21 October 2015

\section{CrossMark}

\author{
${ }^{1}$ Centre for \\ Pharmacoepidemiology and \\ Drug Safety, Manchester \\ Pharmacy School, University \\ of Manchester, Manchester, \\ UK \\ ${ }^{2}$ NIHR Greater Manchester \\ Primary Care Patient Safety \\ Translational Research \\ Centre, University of \\ Manchester, Manchester \\ Academic Health Sciences \\ Centre (MAHSC), \\ Manchester, UK \\ ${ }^{3}$ Centre for Suicide \\ Prevention, Centre for Mental \\ Health and Safety, Institute of \\ Brain, Behaviour and Mental \\ Health, University of \\ Manchester, Manchester, UK
}

Correspondence to Hayley C Gorton; hayley.gorton@postgrad. manchester.ac.uk

\section{ABSTRACT}

Objectives: To establish which non-psychotropic medications have been assessed in relation to risk of suicide or attempted suicide in observational studies, document reported associations and consider study strengths and limitations.

Design: Systematic review.

Methods: Four databases (Embase, Medline, PsycINFO and International Pharmaceutical Abstracts) were searched from 1990 to June 2014, and reference lists of included articles were hand-searched. Casecontrol, cohort and case only studies which reported suicide or attempted suicide in association with any non-psychotropic medication were included.

Outcome measures: The outcomes eligible for inclusion were suicide and attempted suicide, as defined by the authors of the included study.

Results: Of 11792 retrieved articles, 19 were eligible for inclusion. Five studies considered cardiovascular medication and antiepileptics; two considered leukotriene receptor antagonists, isotretinoin and corticosteroids; one assessed antibiotics and another assessed varenicline. An additional study compared multiple medications prescribed to suicide cases versus controls. There was marked heterogeneity in study design, outcome and exposure classification, and control for confounding factors; particularly comorbid mental and physical illness. No increased risk was associated with cardiovascular medications, but associations with other medications remained inconclusive and meta-analysis was inappropriate due to study heterogeneity.

Conclusions: Whether non-psychotropic medications are associated with increased risk of suicide or attempted suicide remains largely unknown. Robust identification of suicide outcomes and control of comorbidities could improve quantification of risk associated with non-psychotropic medication, beyond that conferred by underlying physical and mental illnesses.

\section{INTRODUCTION}

Worldwide, approximately 800000 people die by suicide annually, ${ }^{1}$ therefore suicide prevention is an international priority. ${ }^{2}$ In addition to being the single strongest

\section{Strengths and limitations of this study}

- To our knowledge, this is the first systematic review to critically evaluate observational studies that have reported suicide and attempted suicide in relation to non-psychotropic medication.

- To reduce misclassification, which is problematic with other broader definitions of suicidality, only suicide and attempted suicide outcomes were considered.

- Study heterogeneity precluded statistical pooling of studies within each group of non-psychotropic medication via meta-analyses.

predictor of suicide, ${ }^{1}$ attempted suicide increases risk of all-cause mortality. ${ }^{3}$ A multitude of factors contribute to raised suicide risk, ${ }^{45}$ in particular the presence of mental illness. ${ }^{6} 7$ Additionally, the elevated risk of suicide associated with physical illnesses is becoming increasingly recognised, ${ }^{7-9}$ albeit to a lesser extent than the risk associated with mental illness. ${ }^{7}$

Although suicide risk differs between physical illnesses, ${ }^{7}$ individuals who have been hospitalised for any physical illnesses are at higher risk of suicide than those who have not. ${ }^{8}$ Various factors may contribute to increased suicide risk, including disease severity, comorbidities and impact on quality of life. Furthermore, it is largely unknown whether the non-psychotropic medications used to treat physical illnesses influence suicide risk beyond that attributed by the illness itself.

In 2009, the US Food and Drug Administration (FDA) required 125 medications, some non-psychotropic, to provide labelled warnings of suicidal ideation or behaviour, or both, in product information. ${ }^{10}$ Suicidality outcomes encompass a broad spectrum of suicidal intent, ranging from passive ideation without active planning to harm oneself, to self-harm without intent to die, to attempted suicide, to death by 
suicide. ${ }^{11}$ More commonly occurring suicidality outcomes are used as proxies for suicide and attempted suicide because randomised controlled trials (RCTs) are greatly underpowered to examine these rare outcomes Furthermore, participants of RCTs are closely monitored and study medication will be stopped if serious outcomes are observed. Therefore, assessment of suicide and attempted suicide in observational studies is essential to examine potential risks posed by non-psychotropic medication independent of the underlying physical illness.

Associations between selected non-psychotropic medications and suicidality have been considered in narrative reviews ${ }^{12-14}$ and one systematic review has focused on antiepileptic drugs (AEDs). ${ }^{15}$ However, to our knowledge, no systematic review which considers the extent of the associations between all non-psychotropic medications and suicide has been published. We therefore aimed to: (1) identify which non-psychotropic medications have been examined in relation to risk of suicide and attempted suicide in observational studies; (2) discern what associations have been reported; and (3) critically evaluate the strengths and limitations of these studies.

\section{METHOD}

\section{Literature search}

Four electronic databases, Embase, Medline, PsycINFO and International Pharmaceutical Abstracts, were independently searched. In all searches, there was a requirement for suicide or suicidal to be in the title or abstract. Terminology was selected to encompass any nonpsychotropic medication. Psychotropic medications exert their main effect on mental symptoms ${ }^{16}$ therefore, nonpsychotropic medication was accepted as medication not primarily prescribed to treat the mental illnesses described in Diagnostic and Statistical Manual $V^{17}$ and operationalised by exclusion of British National Formulary 68 categories 4.1-4.4, 4.10.1, 4.10.3 and 4.11. ${ }^{18}$ Medication search terms, medical subject headings and explode features were tailored for each database, and required presence in titles or abstracts. The following initial search strategy was used in Embase: suicide or suicidal (ti, ab) AND medicine (ti. ab.) OR medicat* (ti. ab.) OR drug therapy (exp., ti.ab.). Retrieved citations were limited to those published in English between 1990 and June 2014, to encompass any stimulated reporting following a case series of reports regarding suicidality published in $1990 .{ }^{19}$ For each medication group identified, additional searches were performed and reference lists of included studies were hand-searched. The full search strategy along with the study protocol is documented in the online supplementary material.

\section{Study inclusion}

One author (HCG) screened studies against inclusion protocol and the other coauthors (DMA, RTW and NK) provided advice where a decision to include/exclude was unclear. Observational studies including cohort, case-control, case-crossover and self-controlled case series analyses, which pertained to any non-psychotropic medications, were eligible for inclusion. The outcomes of interest were suicide and attempted suicide presented separately or as a combined outcome. Other suicidality outcomes, including suicidal ideation, were excluded. Where authors indicated that the outcomes of interest were analysed separately, but outcomes were published only in combination with other suicidality outcomes, personal contact with these authors was made. Case reports, case series, cross-sectional studies, and RCTs were excluded. Any comparison treatment was permitted. Individuals with psychiatric illness were included providing the cohort was not defined by presence of this illness. This is because symptomatic improvement of the mental illness by medication used to treat the illness may preclude detection of any induction of suicidality and prevent equivalent comparison with nonpsychotropic use. It was expected that AEDs would be a group of medicine retrieved by the literature search. This group does not feature as a class of clinical psychotropic medication per se, but some AEDs would also be classified as mood stabilisers, which are considered psychotropic. ${ }^{16}$ To avoid misrepresentation of the scope of non-psychotropic medication investigated in relation to suicide, we included AEDs in this systematic review. However, any study which focused on the use of AEDs exclusively as mood stabilisers was excluded.

\section{Study analysis}

Study characteristics, key findings (eg, odds ratios, relative risks) and a critical appraisal, including an assessment of bias, are reported for each study in accordance with the Preferred Reporting Items for Systematic Reviews and Meta-analyses (PRISMA) statement. $^{20}$ Studies of all quality levels were included in the review and were critiqued by one author (HCG) and discussed at length with co-authors (DMA and RTW). Existing quality assessment tools do not specifically relate to pharmacoepidemiological studies therefore, the criteria outlined in Neyarapally et $a l \mathrm{~s}^{21}$ quality assessment framework was used to guide the critical evaluation.

\section{RESULTS}

From 11792 retrieved articles, 19 empirical studies ( figure 1) satisfied our inclusion criteria. A primary focus on psychotropic medication, non-relevant outcomes or alternative study design, accounted for the majority of exclusions. Of the included studies (table 1) five studies each explored $\mathrm{AEDs}^{22-26}$ and cardiovascular medications ${ }^{27-31}$ two studies each considered leukotriene receptor antagonists (LTRAs), ${ }^{32} 33$ isotretinoin, ${ }^{34} 35$ and corticosteroids; $;^{36}{ }^{37}$ and one each assessed antibiotics ${ }^{38}$ and varenicline. $^{39}$ One additional study compared various medications used by individuals who died by suicide, to those used by age and sex-matched controls. ${ }^{40}$ 
Figure 1 Flow diagram of included studies.

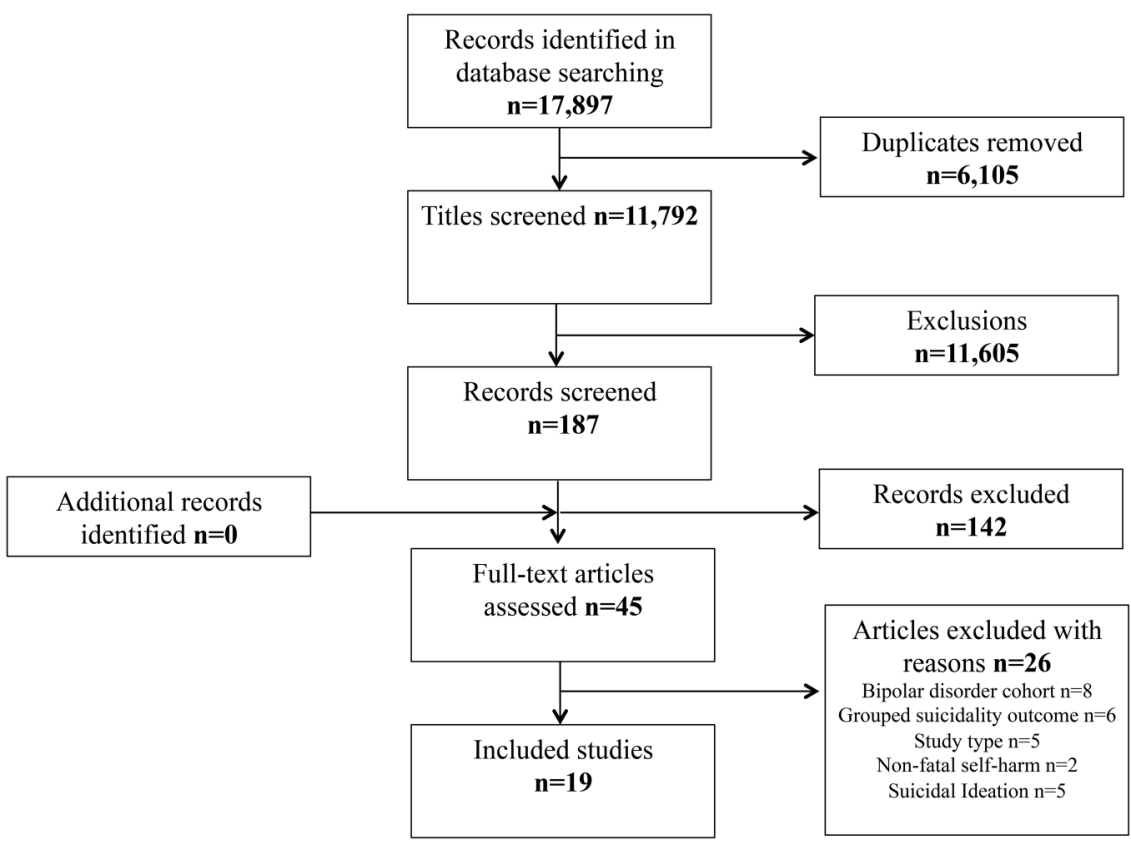

Nine studies reported suicide, ${ }^{24} \quad 25 \quad 27-32 \quad 40$ three reported attempted suicide, ${ }^{23} 33$ two studies presented outcomes separately ${ }^{35} 38$ and five of them combined these outcomes. $^{22} 26343637$ Some studies linked suicide cases to national ${ }^{24-27} 29-31$ or local ${ }^{40}$ mortality data, and others relied on database coding of suicide and attempted suicide. $^{22} \quad 23 \quad 28 \quad 32-34 \quad 36-39$ Studies were conducted in the $\mathrm{UK}^{22} \quad 28 \quad 32 \quad 36-38$ USA, ${ }^{23} \quad 26 \quad 33 \quad 39$ Canada $^{34} 40$ and Scandinavia, $24252729-3135$ and therefore were subject to suicide recording conventions adopted by each country. Population sources included healthcare databases which recorded drugs prescribed, ${ }^{22} \quad 28 \quad 32 \quad 36-38$ dispensed, ${ }^{27} \quad 30 \quad 31$ or both; $^{23} 25262933 \quad 343940$ and hospital inpatient ${ }^{24}$ or specialist registries. $^{35}$

Thirteen studies accounted for psychiatric comorbidities to various extents. ${ }^{22} 23$ 25-2933 3436383940 Statistical adjustment was the most commonly used method. ${ }^{23} 25$ 26-28 $33 \quad 363840$ Exclusion of patients with history of depression ${ }^{29}$ or suicide attempt, ${ }^{22}{ }^{26}$ stratification by psychiatric history ${ }^{34}$ and propensity score matching $^{26} 39$ were also used. In the studies which attempted to mitigate confounding by indication, medication use was restricted to particular conditions, ${ }^{24} 28$ 32-35 stratified by condition 222336 or adjustment for physical illness was performed. $^{22} 252640$ Some studies quantified suicide risk by comparison of a treated group with an untreated group, ${ }^{22} 272936$ the general population ${ }^{31}$ or a group using other medications relevant to that condition. ${ }^{26} 3439$ Use of an individual as their own control in case-only designs ${ }^{25} 3537$ relinquished the need for a separate comparator group.

\section{Antiepileptic drugs}

Of the five studies which investigated AEDs, two reported suicide, ${ }^{24} 25$ one estimated risk of attempted suicide $^{23}$ and two combined both outcomes. ${ }^{22} 26$ Four studies utilised a cohort design, ${ }^{22} 232526$ one of which also performed a case-crossover analysis. ${ }^{25}$ Additionally, Arana et $a l^{22}$ utilised a case-control study, the only design used by Nilsson et $a l^{24}$ Some studies considered individual AEDs, ${ }^{23} 2526$ whereas others assessed all AEDs combined. $^{22} 24$ Comparisons were made with nonexposure, $^{22}$ particular AEDs $^{25}{ }^{26}$ or multiple AEDs compared to monotherapy in individuals diagnosed with epilepsy. $^{24}$

The association between AEDs and suicide remains undetermined and varies between individual AEDs. Arana et al reported an increased risk of suicide and attempted suicide when AEDs were used for conditions other than epilepsy, bipolar disorder or depression; compared to controls who did not receive AEDs nor had these diagnoses (OR 2.57 (95\% CI 1.78 to 3.71)). Conversely, within the epilepsy strata, a reduced risk was identified in the treated group compared to the untreated group (OR $0.59 \quad(95 \%$ CI 0.35 to $0.98)) .^{22}$

Three studies considered risk attributed to individual AEDs. Patorno et $a l^{26}$ suggested an increased risk of suicide and attempted suicide associated with gabapentin compared to topiramate (HR 1.42 (95\% CI 1.11 to 1.80)). ${ }^{26}$ Conversely, both Gibbons et al (2010) ${ }^{23}$ and Olesen et $a t^{25}$ reported no statistically significant difference in suicide attempt rate before or after gabapentin initiation. Patorno et $a l^{26}$ reported an increased risk of suicide and attempted suicide with valproate and lamotrigine compared to topiramate. However, when compared to carbamazepine in a cohort of people with epilepsy, no elevation in risk was identified whereas Olesen $e t a l^{25}$ suggested an increased risk of suicide for the same AEDs, when indication was not restricted. 


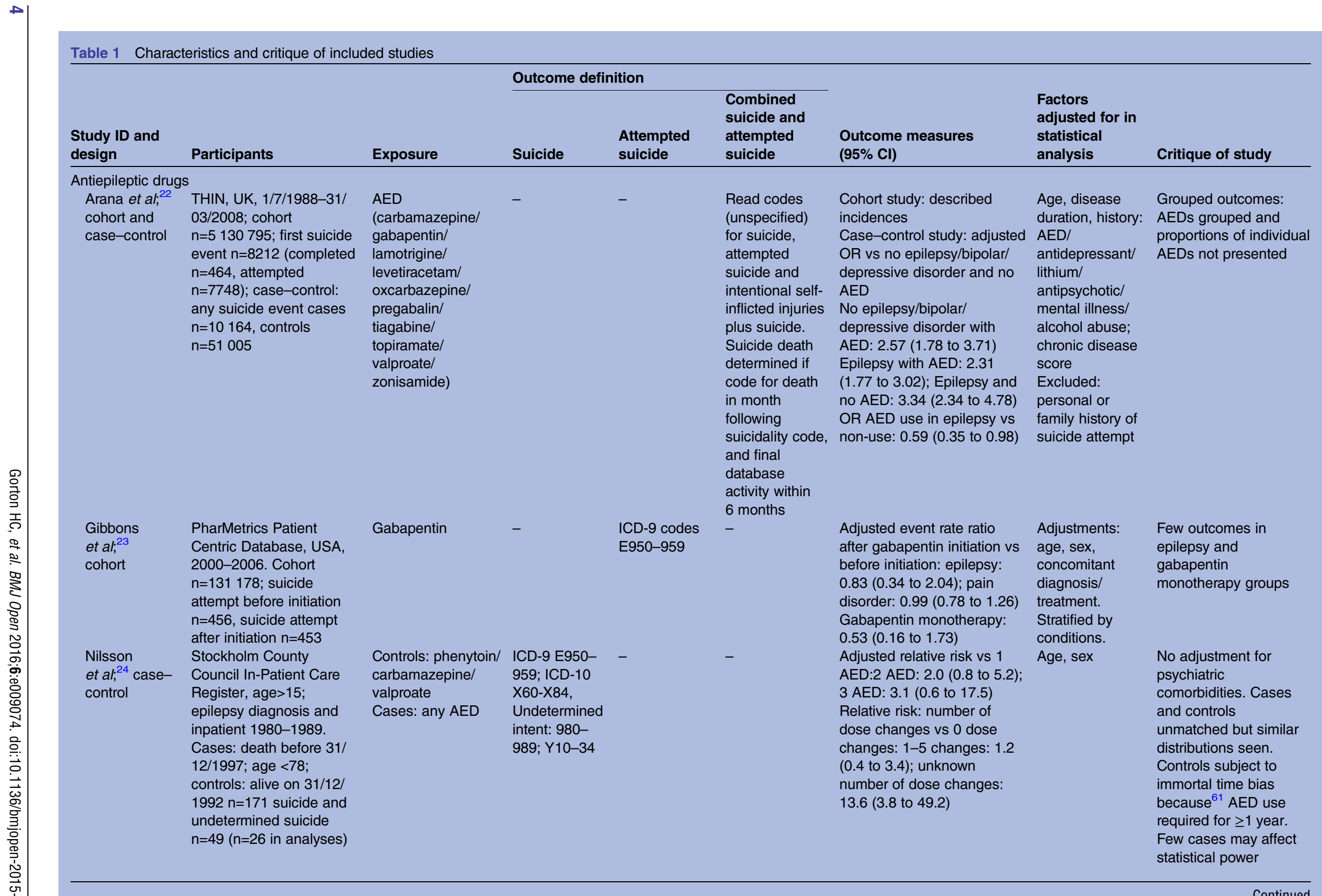

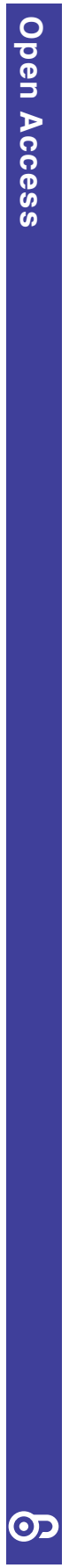

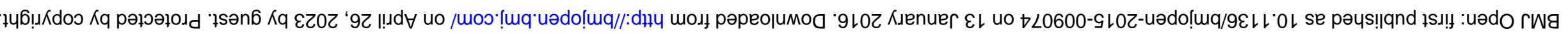




\begin{tabular}{|c|c|c|c|c|c|c|c|c|}
\hline \multirow[b]{2}{*}{$\begin{array}{l}\text { Study ID and } \\
\text { design }\end{array}$} & \multirow[b]{2}{*}{ Participants } & \multirow[b]{2}{*}{ Exposure } & \multicolumn{3}{|c|}{ Outcome definition } & \multirow[b]{2}{*}{$\begin{array}{l}\text { Outcome measures } \\
(95 \% \mathrm{Cl})\end{array}$} & \multirow[b]{2}{*}{$\begin{array}{l}\text { Factors } \\
\text { adjusted for in } \\
\text { statistical } \\
\text { analysis }\end{array}$} & \multirow[b]{2}{*}{ Critique of study } \\
\hline & & & Suicide & $\begin{array}{l}\text { Attempted } \\
\text { suicide }\end{array}$ & $\begin{array}{l}\text { Combined } \\
\text { suicide and } \\
\text { attempted } \\
\text { suicide }\end{array}$ & & & \\
\hline $\begin{array}{l}\text { Olesen et al; }{ }^{25} \\
\text { case- } \\
\text { crossover } \\
\text { analyses and } \\
\text { cohort }\end{array}$ & $\begin{array}{l}\text { Danish databases: } \\
\text { National Prescription } \\
\text { Register, Danish National } \\
\text { Patient Register and } \\
\text { National Causes of Death } \\
\text { Registry } 1 / 1 / 1997-31 / 12 / \\
2006 \text {, age } \geq 10 \text {. } \\
\text { Case-crossover: suicide } \\
\text { total } n=6780 \text {, in study } \\
\text { period } n=898 \text {. Cohort: } \\
\text { newly prescribed AED } \\
n=169725 \text {, Suicide } n=670 \\
\text { during treatment } n=268\end{array}$ & $\begin{array}{l}\text { AED } \\
\text { (carbamazepine/ } \\
\text { clobazam/ } \\
\text { clonazepam/ } \\
\text { gabapentin/ } \\
\text { lamotrigine/ } \\
\text { levetiracetam/ } \\
\text { oxcarbazepine/ } \\
\text { phenobarbital/ } \\
\text { phenytoin/ } \\
\text { pregabalin/ } \\
\text { primidone/ } \\
\text { tiagabine/ } \\
\text { topiramate/ } \\
\text { valproate// } \\
\text { zonisamide) }\end{array}$ & $\begin{array}{l}\text { National } \\
\text { Cause of } \\
\text { Death } \\
\text { Register: } \\
\text { ICD-10 } \\
\text { X60-X84 }\end{array}$ & - & - & 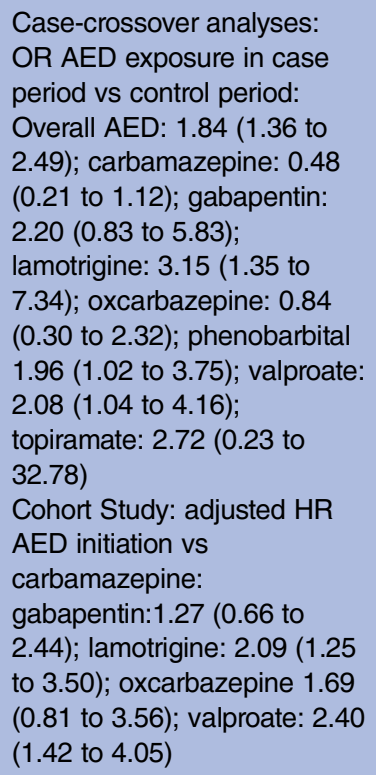 & $\begin{array}{l}\text { Cohort analyses: } \\
\text { age, sex, } \\
\text { socioeconomic } \\
\text { status, } \\
\text { Charlsons' } \\
\text { score, civil } \\
\text { status, epilepsy/ } \\
\text { psychiatric } \\
\text { disorder, opiate } \\
<180 \text { days prior } \\
\text { to index date; } \\
\text { concomitant } \\
\text { antidepressant/ } \\
\text { antipsychotic/ } \\
\text { anxiolytic }\end{array}$ & $\begin{array}{l}\text { Case-crossover design } \\
\text { suitability: Exposure } \\
\text { may be influenced by } \\
\text { indication which may } \\
\text { independently increase } \\
\text { risk of suicide when } \\
\text { exposed to treatment }\end{array}$ \\
\hline $\begin{array}{l}\text { Patorno } \\
\text { et } a l{ }_{;}^{26} \text { cohort }\end{array}$ & $\begin{array}{l}\text { HealthCore Integrated } \\
\text { Research Database, USA; } \\
07 / 2001-12 / 2006 \text {, age } \\
\geq 15 \text {; new AED } \\
\text { Cohort } n=297620 \text { new } \\
\text { treatment episodes, } \\
\text { Suicide } n=26 \text {, Suicide } \\
\text { Attempt } n=801\end{array}$ & $\begin{array}{l}\text { New exposure to } \\
\text { AED } \\
\text { (carbamazepine/ } \\
\text { ethosuxamide/ } \\
\text { felbamate/ } \\
\text { gabapentin/ } \\
\text { lamotrigine/ } \\
\text { levetiracetam/ } \\
\text { oxcarbazepine/ } \\
\text { phenobarbital } \\
\text { phenytoin/ } \\
\text { pregabalin/ } \\
\text { primidone/ } \\
\text { tiagabine/ } \\
\text { topiramate/ } \\
\text { valproate/ } \\
\text { zonisamide) }\end{array}$ & - & - & $\begin{array}{l}\text { Suicide } \\
\text { attempt: } \\
\text { emergency } \\
\text { department/ } \\
\text { hospitalisation } \\
\text { ICD-9 } \\
\text { E950-E958 } \\
\text { Suicide: ICD-10 } \\
\text { X60-84 }\end{array}$ & $\begin{array}{l}\text { Adjusted HR for suicide and } \\
\text { suicide attempt within } \\
180 \text { days of exposure vs } \\
\text { topiramate: carbamazepine: } \\
1.24 \text { (0.77 to } 1.99) \text {; } \\
\text { gabapentin: } 1.42 \text { ( } 1.11 \text { to } \\
1.80 \text { ); lamotrigine: } 1.84 \text { ( } 1.43 \\
\text { to } 2.37) \text {; levetricetam: } 1.63 \\
\text { ( } 0.84 \text { to } 3.16) ; \\
\text { oxcarbazepine: } 2.07 \text { ( } 1.52 \text { to } \\
2.80 \text { ); valproate: } 1.65 \text { ( } 1.25 \\
\text { to } 2.19 \text { ) } \\
\text { Propensity score matched } \\
\text { HR for suicide or suicide } \\
\text { attempt in epilepsy/seizure } \\
\text { disorder stratum vs } \\
\text { carbamazepine: gabapentin: } \\
13.92 \text { (1.82 to } 106.38 \text { ); } \\
\text { oxcarbazepine: } 0.73 \text { ( } 0.16 \text { to } \\
3.28 \text { ); phenytoin: } 3.48 \text { ( } 0.97 \\
\text { to } 12.47) \text {; topiramate: } 0.67 \\
\text { (0.37 to } 1.19 \text { ); valproate: } \\
0.49 \text { (0.09 to } 2.70 \text { ) }\end{array}$ & $\begin{array}{l}49 \text { covariates } \\
\text { including } \\
\text { diagnosis of or } \\
\text { medication for: } \\
\text { depression, } \\
\text { mania, } \\
\text { psychosis, } \\
\text { anxiety, } \\
\text { substance/ } \\
\text { alcohol abuse, } \\
\text { personality } \\
\text { disorder, other } \\
\text { psychiatric } \\
\text { disorders, } \\
\text { physical } \\
\text { disorders } \\
\text { Propensity score } \\
\text { matched } \\
\text { analysis: in } \\
\text { sensitivity } \\
\text { analysis }\end{array}$ & $\begin{array}{l}\text { Comparator suitability: } \\
\text { topiramate selected but } \\
\text { low frequency of use in } \\
\text { epilepsy, although } \\
\text { comparison with } \\
\text { carbamazepine was } \\
\text { repeated for this } \\
\text { stratum. } \\
\text { Imprecise estimate for } \\
\text { gabapentin when } \\
\text { restricted to people with } \\
\text { epilepsy/seizure } \\
\text { disorder: requires } \\
\text { cautious interpretation }\end{array}$ \\
\hline
\end{tabular}




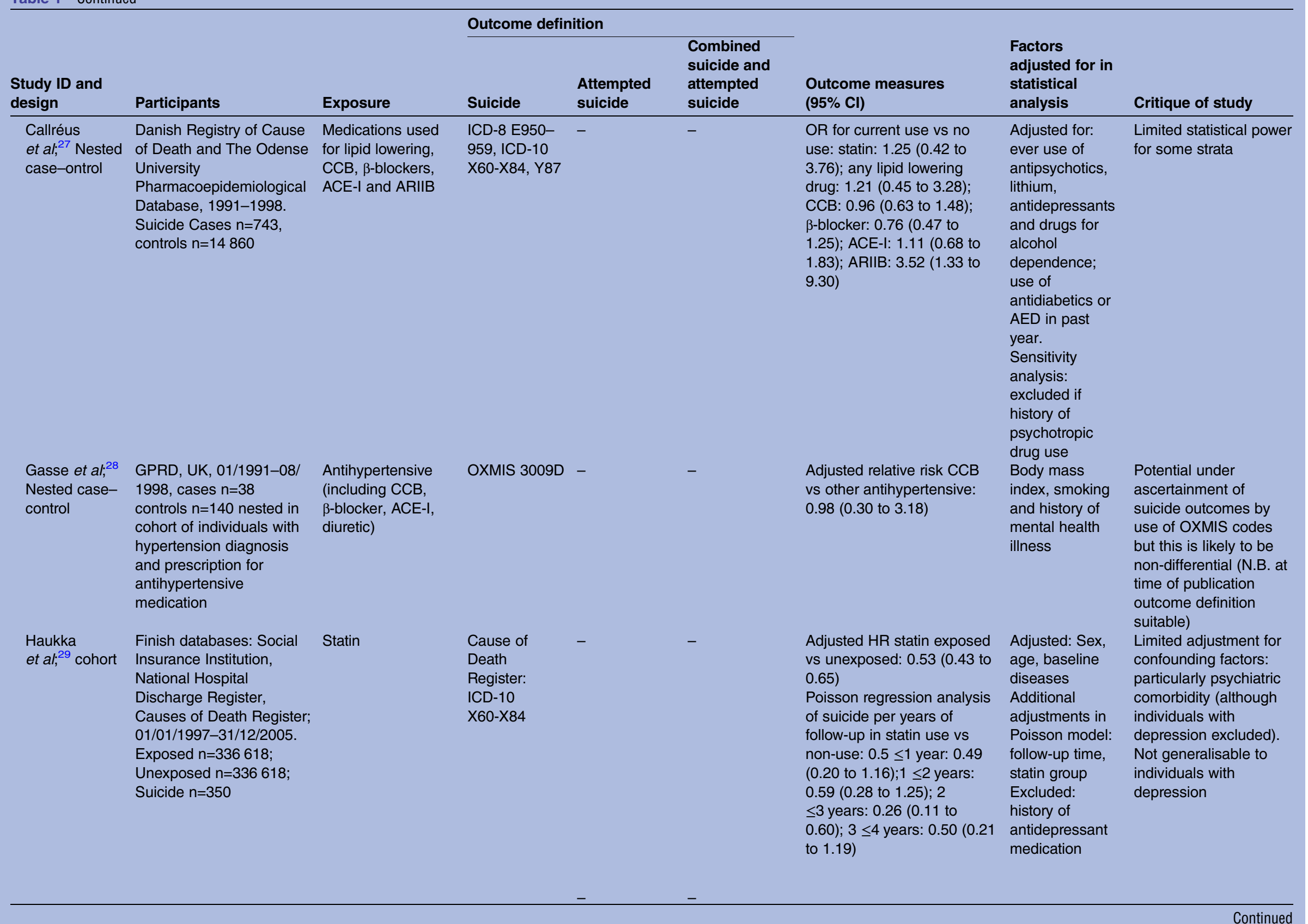


Outcome definition

\begin{tabular}{|c|c|c|c|}
\hline $\begin{array}{l}\text { Study ID and } \\
\text { design }\end{array}$ & Participants & Exposure & Suicide \\
\hline $\begin{array}{l}\text { Lindberg } \\
\text { et } a l^{30} \text { cohort }\end{array}$ & $\begin{array}{l}\text { Swedish pharmacy } \\
\text { data,1988-1989, cohort } \\
n=3397 ; \text { CCB } n=617 \text {, } \\
\text { other cardiovascular } \\
\text { medication } n=2780\end{array}$ & $\begin{array}{l}\text { Cardiovascular } \\
\text { medication } \\
\text { including CCB, } \\
\beta \text {-blocker, ACE I, } \\
\text { diuretic }\end{array}$ & $\begin{array}{l}\text { Swedish } \\
\text { Mortality } \\
\text { Register: } \\
\text { ICD-9 E950- } \\
\text { 959, E980- } \\
\text { 989 }\end{array}$ \\
\hline $\begin{array}{l}\text { Sørensen } \\
\text { et } a l ;{ }^{31} \text { cohort }\end{array}$ & $\begin{array}{l}\text { Pharmacoepidemiological } \\
\text { Prescription database of } \\
\text { North Jutland County, } \\
\text { Denmark, 01/01/1989-31/ } \\
\text { 12/1995; cohort } n=58529\end{array}$ & $\begin{array}{l}\text { CCB, } \beta \text {-blocker or } \\
\text { ACE inhibitor }\end{array}$ & $\begin{array}{l}\text { National } \\
\text { Death } \\
\text { Certificate } \\
\text { Files: ICD 8/ } \\
10 \text { codes }\end{array}$ \\
\hline
\end{tabular}

12/1995; cohort $n=58529$

10 codes

Leukotriene receptor antagonists

\begin{tabular}{|c|c|c|c|c|c|c|}
\hline $\begin{array}{l}\text { Jick et } a l^{32} \\
\text { cohort }\end{array}$ & $\begin{array}{l}\text { GPRD, UK, 02/1998-03/ } \\
\text { 2007; cohort } n=23500\end{array}$ & Montelukast & $\begin{array}{l}\text { Computer } \\
\text { recorded } \\
\text { diagnosis }\end{array}$ & - & - & $\begin{array}{l}\text { Suicides in } 21050 \text { person } \\
\text { years } n=0\end{array}$ \\
\hline
\end{tabular}

Schumock LifeLink Health Plan

et $a l^{33}$ Nested Claims Database, USA,

Montelukast, asthma and new

an asthma treatment; age 5-24. Cases $n=344$ Controls $n=3438$ E950-E959

zafirlukast, zileuton

E950-E959

ICD-9 code

\section{Combined}

Attempted attempted suicide suicide

$(95 \% \mathrm{Cl})$

7-year suicide risk difference

for CCB use vs non-use:

$7.5 / 1000$ person-years

( $\mathrm{p}=0.002)$

Adjusted relative risk: 5.4

(1.4 to 20.5)

SMR ever-use: $\beta$-blocker:

1.6 (1.2 to 2.1); CCB: 1.2

(0.8 to 1.7); ACE-I 1.2 (0.7

to 1.8$)$. SMR present use of

single study drug: $\beta$-blocker:

1.4 (0.9 to 2.1); CCB: 1.2

(0.7 to 1.9$) ; 1.2$ (0.5 to 2.4 ).

SMR for $\beta$-blocker use only

(present and former): low

lipid solubility: 0.9 (0.4 to

1.9); high lipid solubility: 2.7

(1.7 to 4.1 )

years $n=0$

Adjusted OR vs never use: current use: 0.70 (0.36 to 1.39), immediate past use: 0.95 (0.36 to 2.50), past use: 0.69 (0.32 to 1.50$)$. Ever use: 0.74 (0.46 to 1.20 )
Factors

adjusted for in

statistical

analysis

Critique of study

ted for Limited statistiontinued

power: 9 suicides in

total.

No adjustment for

many confounders:

including history of

mental illness.

Indication unknown

Comparison to general population: differences

in baseline

characteristics and

adjustment for

confounders not

possible (although

some potential

confounding factors

compared between

treatment groups).

Potential overestimation

of exposure: present

use considered for

180 days after

prescription received

No comparison group-would be

necessary to compare incidence if cases were identified

History: bipolar All cases were exposed disorder, depression,

other mental

disorder,

substance

abuse, suicide

to montelukast rather

than other drugs in this

class (reflects

prescribing trends), but

results attributed to

entire class

psychological

counselling;

asthma severity

(by proxy) 


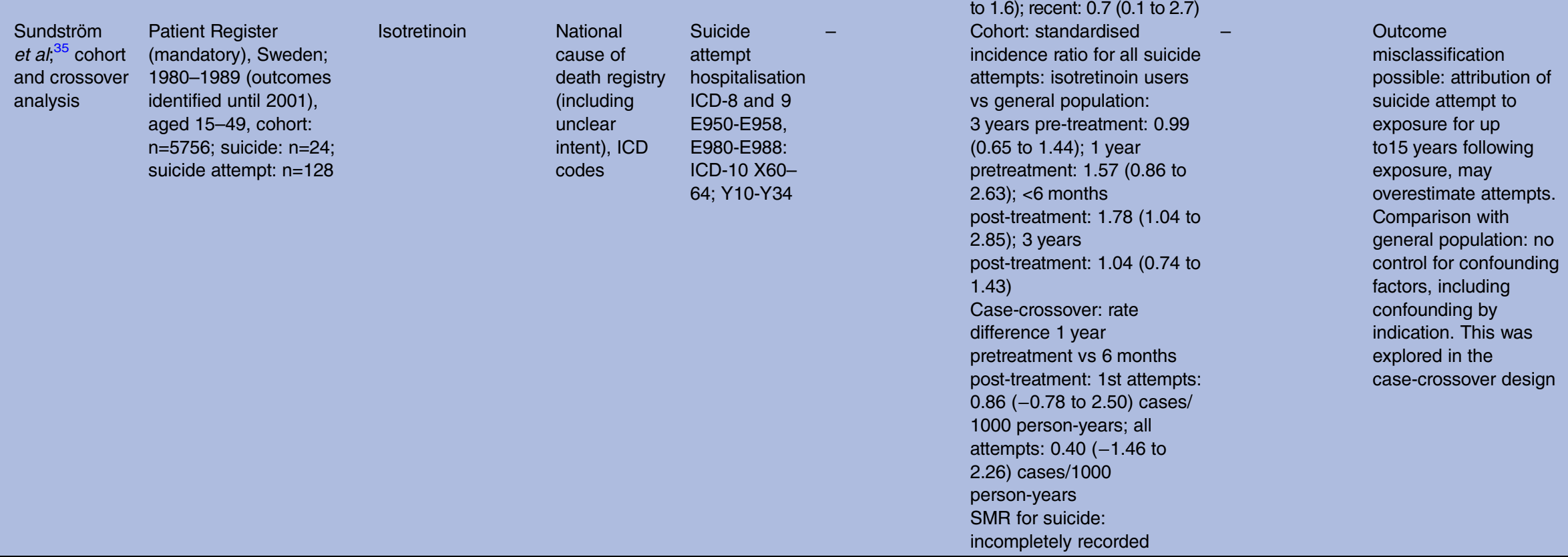




\begin{tabular}{|c|c|c|c|c|c|c|c|c|}
\hline \multirow[b]{2}{*}{$\begin{array}{l}\text { Study ID and } \\
\text { design }\end{array}$} & \multirow[b]{2}{*}{ Participants } & \multirow[b]{2}{*}{ Exposure } & \multicolumn{3}{|c|}{ Outcome definition } & \multirow[b]{2}{*}{$\begin{array}{l}\text { Outcome measures } \\
(95 \% \mathrm{Cl})\end{array}$} & \multirow[b]{2}{*}{$\begin{array}{l}\text { Factors } \\
\text { adjusted for in } \\
\text { statistical } \\
\text { analysis }\end{array}$} & \multirow[b]{2}{*}{ Critique of study } \\
\hline & & & Suicide & $\begin{array}{l}\text { Attempted } \\
\text { suicide }\end{array}$ & $\begin{array}{l}\text { Combined } \\
\text { suicide and } \\
\text { attempted } \\
\text { suicide } \\
\end{array}$ & & & \\
\hline $\begin{array}{l}\text { Fardet et } a{ }^{36}{ }^{36} \\
\text { cohort }\end{array}$ & $\begin{array}{l}\text { THIN, UK, 01/01/1990- } \\
\text { 31/12/2008; age } \geq 18 ; \text { new } \\
\text { glucocorticoid exposure } \\
n=372696 ; \text { exposed with } \\
\text { indication } n=261272 ; \\
\text { unexposed } n=1224984 ; \\
\text { unexposed matched by } \\
\text { indication } n=660776 ; \\
\text { exposed groups suicide } \\
n=19 ; \text { suicide attempt } \\
n=90\end{array}$ & $\begin{array}{l}\text { Oral glucocorticoid } \\
\text { (betamethasone/ } \\
\text { deflazacort/ } \\
\text { dexamethasone/ } \\
\text { hydrocortisone/ } \\
\text { methylprednisolone/ } \\
\text { prednisone/ } \\
\text { prednisolone/ } \\
\text { triamcinolone/) }\end{array}$ & - & - & $\begin{array}{l}\text { Read codes } \\
\text { and } \\
\text { cross-searched } \\
\text { death } \\
\text { certificates }\end{array}$ & $\begin{array}{l}\text { Adjusted HR: exposed vs } \\
\text { unexposed: } 5.27 \text { ( } 3.82 \text { to } \\
7.29 \text { ) } \\
\text { Exposed vs unexposed, } \\
\text { matched by indication: } 6.89 \\
\text { (4.52 to } 10.50)\end{array}$ & $\begin{array}{l}\text { Adjusted: age, } \\
\text { sex, history } \\
\text { neuropsychiatric } \\
\text { disorder } \\
\text { Separate } \\
\text { analysis for } \\
\text { cohorts matched } \\
\text { by indication }\end{array}$ & $\begin{array}{l}\text { Handling of repeated } \\
\text { courses: random } \\
\text { selection of course } \\
\text { could alter baseline risk } \\
\text { dependent on course } \\
\text { selected. If later } \\
\text { courses chosen, } \\
\text { individual subject to } \\
\text { immortal time }{ }^{61} \text { bias } \\
\text { until this time }\end{array}$ \\
\hline $\begin{array}{l}\text { Fardet et al, }{ }^{37} \\
\text { cohort and } \\
\text { self-controlled } \\
\text { case series }\end{array}$ & $\begin{array}{l}\text { THIN, UK 01/01/1990-31/ } \\
\text { 12/2008; age } \geq 18 \text {; } \\
\text { glucocorticoid use for } \\
1-3 \text { years. Cohort: } \\
n=21 \text { 995. Eligible for } \\
\text { self-controlled case series } \\
\text { analysis: } n=991 ; \text { suicide } \\
\text { or suicide attempt } n=6\end{array}$ & $\begin{array}{l}\text { Oral glucocorticoid } \\
\text { (betamethasone/ } \\
\text { deflazacort/ } \\
\text { dexamethasone/ } \\
\text { hydrocortisone/ } \\
\text { methylprednisolone/ } \\
\text { prednisone/ } \\
\text { prednisolone/ } \\
\text { triamcinolone/) }\end{array}$ & - & - & Read codes & $\begin{array}{l}\text { Cohort: incidence rate for } \\
\text { suicide or attempted suicide } \\
\text { during withdrawal period: } \\
0.03(0.01 \text { to } 0.2) \\
\text { Self-controlled case series: } \\
\text { incident rate ratio in } \\
\text { withdrawal period vs ref. } \\
\text { period: } 0.62 \text { ( } 0.06 \text { to } 6.92) \\
\text { (ref. period: } 5-3 \text { months prior } \\
\text { to discontinuation) }\end{array}$ & & $\begin{array}{l}\text { Inadequate statistical } \\
\text { power: only } 6 \text { cases of } \\
\text { suicide or attempted } \\
\text { suicide. } \\
\text { Potential immortal time } \\
\text { bias: }^{61} \text { for entry into the } \\
\text { cohort, must not have } \\
\text { died in first year of } \\
\text { glucocorticoid use. To } \\
\text { be eligible for the } \\
\text { self-controlled case } \\
\text { series analysis, any } \\
\text { suicides must occur in } \\
\text { the withdrawal period }\end{array}$ \\
\hline \multicolumn{9}{|c|}{ Antibiotics (quinolones) } \\
\hline $\begin{array}{l}\text { Jick et } a l^{38} \\
\text { Nested ase } \\
\text { Control }\end{array}$ & $\begin{array}{l}\text { GPRD, UK, ever exposed } \\
\text { to quinolone, 01/01/1991- } \\
\text { 30/4/1995, age } 15-84 \text {. } \\
\text { Cases } n=348 \text { (suicide } \\
n=13 \text {, suicide attempt } \\
n=206 \text { suicidal ideation } \\
n=129 \text { ) Control } n=808 \\
\text { (NB. Outcomes analysed } \\
\text { separately) }\end{array}$ & $\begin{array}{l}\text { Quinolone or other } \\
\text { antibiotic in } 1-30 \text { or } \\
31-180 \text { days prior } \\
\text { to index date }\end{array}$ & $\begin{array}{l}\text { OXMIS code } \\
\text { 3009D }\end{array}$ & $\begin{array}{l}\text { OXMIS code } \\
\text { L3009P, } \\
9779 \text { L, 3009C }\end{array}$ & - & $\begin{array}{l}\text { Adjusted relative risk } \\
\text { estimate for suicide attempt } \\
\text { vs non-exposure: quinolone } \\
1-30 \text { days: N/A; quinolone } \\
31-180 \text { days: } 0.6(0.2 \text { to } \\
1.5) \text {; other antibiotic } 1- \\
30 \text { days: } 1.2(0.5 \text { to } 2.6) \text {; } \\
\text { other antibiotic } 31-180 \text { days: } \\
0.9 \text { ( } 0.5 \text { to } 1.5)\end{array}$ & $\begin{array}{l}\text { Age, sex, history } \\
\text { of: depression, } \\
\text { suicidal } \\
\text { behaviour, } \\
\text { insomnia, } \\
\text { psychosis, } \\
\text { anxiety, } \\
\text { alcoholism and } \\
\text { epilepsy }\end{array}$ & $\begin{array}{l}\text { Inadequate statistical } \\
\text { power: precluded } \\
\text { calculation of risk within } \\
\text { first month of quinolone } \\
\text { exposure or risk of } \\
\text { suicide death. } \\
\text { Possible } \\
\text { underestimation of } \\
\text { suicide and attempted } \\
\text { suicide: because 1st } \\
\text { recorded event used in } \\
\text { multiple outcomes. } \\
\text { However, all suicidal } \\
\text { ideation comparisons } \\
\text { were non-significant }\end{array}$ \\
\hline
\end{tabular}

$31 / 12 / 2008$; age $\geq 18$; new

glucocorticoid exposure

dication $n=261272$;

xposed groups suicide

Fardet et $a l^{37}$

(betamethasone/

deflazacort

$=21$ 995. Eligible for

methylprednisolone/

triamcinolone/)

were non-significant

Continued 
Table 1 Continued

\begin{tabular}{|c|c|c|c|c|c|c|c|c|}
\hline \multirow[b]{2}{*}{$\begin{array}{l}\text { Study ID and } \\
\text { design }\end{array}$} & \multirow[b]{2}{*}{ Participants } & \multirow[b]{2}{*}{ Exposure } & \multicolumn{3}{|c|}{ Outcome definition } & \multirow[b]{2}{*}{$\begin{array}{l}\text { Outcome measures } \\
(95 \% \mathrm{Cl})\end{array}$} & \multirow[b]{2}{*}{$\begin{array}{l}\text { Factors } \\
\text { adjusted for in } \\
\text { statistical } \\
\text { analysis }\end{array}$} & \multirow[b]{2}{*}{ Critique of study } \\
\hline & & & Suicide & $\begin{array}{l}\text { Attempted } \\
\text { suicide }\end{array}$ & $\begin{array}{l}\text { Combined } \\
\text { suicide and } \\
\text { attempted } \\
\text { suicide } \\
\end{array}$ & & & \\
\hline $\begin{array}{l}\text { Gibbons and } \\
\text { Mann; } 39 \\
\text { cohort }\end{array}$ & $\begin{array}{l}\text { Military Healthcare } \\
\text { System, USA, 01/08/ } \\
\text { 2006-31/08/2007. Cohort } \\
\text { treated with varenicline } \\
(n=19933) \text {; NRT patches } \\
\text { ( } n=15 \text { 867). After } \\
\text { matching by propensity } \\
\text { score: patients } n=26430 \text {; } \\
\text { included suicide attempts } \\
n=5\end{array}$ & $\begin{array}{l}\text { Varenicline or NRT } \\
\text { patches }\end{array}$ & - & $\begin{array}{l}\text { ICD-9 E950- } \\
959\end{array}$ & - & $\begin{array}{l}\text { OR varenicline vs NRT in } \\
\text { patients for whom propensity } \\
\text { score matching was } \\
\text { possible: } 0.67 \text { ( } 0.11 \text { to } 3.99)\end{array}$ & $\begin{array}{l}\text { Propensity score } \\
\text { matching: age, } \\
\text { marital status, } \\
\text { race, sex, } \\
\text { Charlsons' } \\
\text { score, inpatient } \\
\text { admissions, } \\
\text { psychiatric } \\
\text { comorbidity, } \\
\text { psychotropic } \\
\text { medication }\end{array}$ & $\begin{array}{l}\text { Limited power: few } \\
\text { suicide attempt events } \\
(n=5)\end{array}$ \\
\hline \multicolumn{9}{|l|}{ Other medication } \\
\hline $\begin{array}{l}\text { Voaklander } \\
\text { et } a l^{40} \text { case- } \\
\text { control }\end{array}$ & $\begin{array}{l}\text { British Columbia Vital } \\
\text { Statistics, Health } \\
\text { Insurance Registration } \\
\text { File, Pharmacare and } \\
\text { Physician Claim File, } \\
\text { Canada, } 1993-2002 \text {, age } \\
\geq 66 \text {, suicide cases } n=602 \\
\text { controls } n=2999\end{array}$ & $\begin{array}{l}\text { anti-diabetic } \\
\text { agents, } \\
\text { anticoagulants, } \\
\text { cardiovascular } \\
\text { drugs, NSAID, ulcer } \\
\text { medication, steroids }\end{array}$ & $\begin{array}{l}\text { ICD-9 } \\
\text { E950-E959; } \\
\text { ICD-10: X60- } \\
\text { X84 }\end{array}$ & - & - & $\begin{array}{l}\text { Unadjusted ORs vs non-use: } \\
\text { antihypertensive medication: } \\
0.94 \text { ( } 0.67 \text { to } 1.31 \text { ); lipid } \\
\text { lowering medication: } 0.60 \\
\text { ( } 0.28 \text { to } 1.26) \text {; } \\
\text { anticoagulants: } 1.07 \text { ( } 0.52 \text { to } \\
2.22) \text {; diuretics: } 0.94 \text { (0.66 to } \\
1.36) \text {; ulcer medication: } 1.88 \\
\text { (1.35 to } 2.62) \text {; steroids: } 1.33 \\
\text { (0.88 to } 2.00) \text {. } \\
\text { Fully adjusted OR: diuretics: } \\
0.49 \text { ( } 0.31 \text { to } 0.76)\end{array}$ & $\begin{array}{l}\text { Fully adjusted } \\
\text { analyses: } \\
\text { demographics, } \\
\text { co-morbidity, } \\
\text { medication use }\end{array}$ & $\begin{array}{l}\text { Fully adjusted analysis } \\
\text { not done for all } \\
\text { medications which } \\
\text { suggested significance } \\
\text { at the unadjusted level } \\
\text { (eg, ulcer medications) }\end{array}$ \\
\hline
\end{tabular}




\section{Cardiovascular medications}

Two nested case-control studies ${ }^{27} 28$ and three cohort studies ${ }^{29-31}$ assessed risk of suicide associated with various cardiovascular medications. In all but one, there was no evidence of association with increased or decreased suicide risk. An initial suggestion of increased risk of suicide with calcium channel blockers (CCB) was made by Lindberg et al but the reported association was questioned due to small sample size and lack of control for confounding factors. ${ }^{41}{ }^{42}$ Subsequent studies dismissed any association with CCB use and suicide. ${ }^{27} 2831$ Similarly, there was no difference in risk for ACE inhibitor or $\beta$-blocker use compared to non-use ${ }^{27}$ during monotherapy versus the general population. ${ }^{31}$ An increased standardised mortality ratio was, however, suggested for highly lipid soluble $\beta$-blockers but attributed in part to use in migraine. ${ }^{31}$

An unexpected increased suicide risk with angiotensin II receptor antagonists was reported by Callréus et $a l^{27}$ (OR 3.52 (95\% CI 1.33 to 9.30) ), despite control of multiple potential confounding factors. However, few suicides were reported (current use $\mathrm{n}=5$ ) and, when controlled for psychiatric history, this association became non-significant. Based on the same number of suicide cases, no association between statins and suicide was made. Corroboratively, Haukka et $a l^{29}$ suggested no increase in suicide risk in statin users versus non-users, in any follow-up time investigated. When cardiovascular medication use was compared to non-use by Voaklander et $a l^{40}$ only diuretics were suggested to significantly influence risk; a protective effect was suggested in the fully adjusted analysis (OR 0.49 (95\% CI 0.31 to 0.76$)$ ).

\section{Leukotriene receptor antagonists}

Two observational studies reported that no increased risk of suicide or attempted suicide was apparent when LTRAs, montelukast, zafirlukast and zileuton, were used for the treatment of asthma. ${ }^{32}{ }^{33}$ No suicides were reported in Jick $e t a l \mathrm{~s}^{32}$ cohort of individuals exposed to montelukast, although one case was retrospectively disqualified based on time lag between exposure and outcome. Similarly, Schumock $e t a l^{33}$ did not detect any difference in suicide attempt during use of any LTRA compared to non-use in their nested case-control study of individuals diagnosed with asthma, aged 5-24 years.

\section{Isotretinoin}

No difference in the combined risk of attempted and completed suicide was associated with isotretinoin or antibiotics, compared to non-exposure, in Jick et al $\mathrm{s}^{34}$ cohort of individuals with acne, regardless of psychiatric history. Similarly, there was no significant difference in attempted suicide risk before treatment compared to 6 months after treatment, in Sundström et al $\mathrm{s}^{35}$ crossover analysis. On the other hand, when compared to the general population, the highest elevated risk was observed in the first 6 months of treatment, but this risk was rising prior to medication initiation.

\section{Corticosteroids}

No difference in suicide risk was associated with steroid use versus non-use by Voaklander et al. ${ }^{40}$ Conversely, in Fardet et als (2012) cohort study, a fivefold increased risk of suicide and attempted suicide was reported following glucocorticoid exposure, compared to non-exposure (HR 5.27 (95\% CI 3.82 to 7.29 )) but incidence was low. ${ }^{36}$ In a subsequent self-controlled case series, no difference was detected during withdrawal period compared to treatment periods, ${ }^{37}$ although this assertion was based on only six cases.

\section{Antibiotics}

In the single nested case-control study which focused on quinolone antibiotics, no difference in risk of attempted suicide was detected following exposure to quinolones or other antibiotics, compared to non-exposure. ${ }^{38}$

\section{Varenicline}

One cohort study reported suicide attempt, separately from other outcomes, in relation to varenicline use. There was no difference in risk during use of varenicline or nicotine replacement patches, when individuals were matched by propensity score. ${ }^{39}$

\section{DISCUSSION}

The primary aims of this systematic review were to establish which groups of non-psychotropic medications have been associated with suicide and attempted suicide in observational epidemiological studies; and to quantify the influence these medications have on this risk, beyond that conferred by underlying illness. Overall, the contribution of corticosteroids, ${ }^{3640}$ isotretinoin $^{34} 35$ and $\mathrm{AEDs}^{22-26}$ to risk of suicide and attempted suicide remains unresolved while there seems no increased risk associated with cardiovascular medications. ${ }^{27-29}{ }^{31}$ Neither the single studies which investigated quinolones $^{38}$ or varenicline, ${ }^{39}$ nor the two which assessed LTRAs, ${ }^{32}{ }^{33}$ suggested an increased risk of suicidality.

With the exception of the cardiovascular medicines, all groups of medications identified in this review have been the subject of FDA or UK Medicines and Healthcare Products Regulatory Agency (MHRA) warnings. In 2008, the FDA warned of an increased risk of suicidal behaviour and ideation during AED use, following a meta-analysis of 199 placebo-controlled RCTs involving 43892 patients. An overall OR of 1.80 (95\% CI 1.24 to 2.66 ) was reported. ${ }^{43}$ This mainly reflected an increased risk of suicidal ideation and attempted suicide because only four suicides in total were reported across almost 200 trials. This emphasises the lack of statistical power of RCTs, and even meta-analysis of numerous trials, for examining an outcome as rare as death by suicide. Concerns were raised from medical and research communities regarding the reliability and impact of this warning. The risk was attributed as a class 
effect despite variation in risk associated with individual AEDs, there was potential for heterogeneity in outcome designation and there was an unexplained differential risk dependent on study location. ${ }^{44} 45$ Our review of the observational work which followed is in corroboration with Ferrer $e t a l \mathrm{~s}^{15}$ review, which considered AEDs for any indication, that the association between dying by suicide and AEDs remains inconclusive.

Varenicline is associated with the highest level of warnings issued by the FDA. It may therefore be surprising that only one study which pertains to varenicline is included in this review. This is because no other observational studies considered only suicide or attempted suicide outcomes. Neither observational studies which considered all self-harm outcomes ${ }^{46}{ }^{47}$ nor a pooled analysis of RCTs which considered all suicidality outcomes suggested an increased risk associated with varenicline, ${ }^{39}$ compared to other smoking cessation treatments or placebo. The FDA black-box warning continues to be challenged by the manufacturers of varenicline, based on a meta-analysis of placebo-controlled RCTs. ${ }^{48}$ The FDA also warns of suicidal behaviour during use of isotretinoin and in 2014, an expert review in the UK could neither confirm nor discount an association of suicide with isotretinoin. ${ }^{49}$ Isotretinoin is one of three nonpsychotropic medications on the list of the top 20 medicines most frequently associated with suicide in UK spontaneous reports. ${ }^{50}$ The other two medications, efavirenz and mefloquine, ${ }^{50}$ carry warnings for suicidal behaviour in their drug monographs. ${ }^{18}$ No observational studies reported suicide or attempted suicide outcomes for these medications. Warnings of psychiatric adverse events, including suicidal behaviour, exist for glucocorticoids in the UK, but this group does not feature in the list of FDA drugs linked with suicidal behaviour. ${ }^{10}$

This systematic review has found considerable heterogeneity among studies, which makes comparisons within and between medication groups difficult and quantitative meta-analysis inappropriate. All of the studies that considered cardiovascular medications reported suicide only. Conversely, there was variation in reported outcomes within classes of other medications. Some studies reported attempted suicide or combined suicide and attempted suicide outcomes, sometimes because suicide outcomes were too rare to enable detection of differences between groups. Furthermore, different comparison groups were chosen. Schumock $e t a l^{33}$ controlled for confounding by indication and disease severity by restricting the comparator cohort to people with asthma who use controller medication. On the other hand, comparator group demographics could not be stipulated when standardised mortality rates were estimated. ${ }^{31}$

It is imperative that the underlying risk posed by physical illness as well as pre-existing mental illness or psychological distress is recognised when interpreting any elevation in risk associated with non-psychotropic medication. This is of particular importance for AEDs because epilepsy has been associated with a twofold increased risk of suicide compared to the general population. ${ }^{51}$ Furthermore, AEDs can be prescribed for a variety of physical and psychiatric conditions including bipolar disorder, which is associated with over a 17 -fold increased risk of suicide. ${ }^{752}$ In this review, articles were excluded if the study population was defined by presence of mental illness, to aid interpretation of possible associations with medication separately from to those conferred by the mental illness. We acknowledge that a limitation of this review is that some studies allowed AED use for any indication, which may have included mental illness. This was, however, accounted for by adjustment for or stratification by mental illness in those studies.

Additionally, medications may be used for alternative indications where first line treatment has failed. This could contribute to the increased suicide risk observed by Arana $e t a l^{22}$ when AEDs were prescribed for conditions other than epilepsy, depression or bipolar disorder, much of which was suspected to be indicated for pain. Similarly, Sørensen $e t a l^{31}$ attributed the increased risk associated with lipid soluble $\beta$-blockers in part to the higher prevalence of migraine in this group. Glucocorticoids are often introduced during disease relapse which could contribute to suicide risk, even when indication is controlled for. ${ }^{36}$ Likewise, the increased suicide risk identified prior to initiation of isotretinoin, could be a factor of acne severity. ${ }^{35}$

Observational studies are essentially useful for demonstrating associations rather than causation, although tentative inferences of causality can be put forward if there is robust evidence of concurrence with Bradford-Hill's criteria. ${ }^{53}$ One of his seven pillars of causality is the biological plausibility of the event, in this case a postulated adverse reaction. Adverse reactions to medication can be denoted as type $\mathrm{A}$, an exaggerated effect of the pharmacology of the medication, or type B, usually an idiosyncratic event, often detected in postmarketing surveillance. ${ }^{54}$ Any observed elevation in risk of suicide could be a consequence of induced depression or occur independently. Potential pathways to suicidality have been suggested for some, but not all, medicines included in this review. Interference with $\gamma$-aminobutyric acid and glutamate may contribute to any observed link between AED usage and elevated suicide risk, ${ }^{55}$ but would differ between individual AEDs. Increased cortisol levels have also been linked to suicide. ${ }^{56}$ Therefore exogenously introduced glucocorticoids could confer similar effects. Reduced lipid levels have also been associated with increased suicide risk. ${ }^{5}$ Conversely, the included studies do not suggest increased risk with lipid-lowering medication use, ${ }^{27} 29$ corroborative with earlier work by Yang et al. ${ }^{57}$

To our knowledge, this is the first systematic review to consider the impact of non-psychotropic medication use on risk of suicide and attempted suicide. Only suicide and attempted suicide outcomes were considered, to minimise outcome misclassification possible when other 
suicidality outcomes are used as proxies. $^{11} 13$ Determination of an individuals' intent to die by suicide is challenging ${ }^{58}{ }^{11}$ therefore other terminologies may incorporate suicide attempt. For example, 'non-fatal selfharm' represents a continuum of suicidal and self-harm behaviours with varying motivations and intentions. To avoid overestimation of outcomes, studies were included only if authors explicitly used the label 'suicide attempt'. This may have precluded inclusion of studies which reported attempted suicide as a composite outcome within another definition of suicidality, and is therefore a limitation of our review. We also acknowledge that screening of studies by a single author introduced the potential for selection bias. In an attempt to reduce this bias, other authors were consulted if selection was unclear and included records were hand-searched for suitable studies, which served as a cross-check.

Inclusion of studies from any country introduced further variation in suicide classification. ${ }^{59}$ In the UK, open verdicts are conventionally included in epidemiological suicide definitions, as most are deemed to be probable suicides that were not designated as such due to the high burden of proof required in coroners' courts. ${ }^{60}$ In other countries, including the USA, open verdicts are generally not included in suicide case definitions. For example, Patorno et al separately reported violent deaths in their US population, up to $87 \%$ of which may be suicides. The trends identified were, however, similar to those for suicide and attempted suicide outcomes. ${ }^{26}$

Determining the cause of any observed increased risk, specifically as a result of mental or physical illness, the medication itself, or a combination of factors, represents a major challenge. Overall assessments are difficult to report due to variation between study outcomes, populations and control for psychiatric and physical comorbidities. Robust identification of suicidality outcomes and control of comorbidities is needed in future observational studies, particularly to investigate suicide risk in association with AEDs, isotretinoin and corticosteroids.

\section{Twitter Follow Hayley Gorton at @hayley_gorton}

Contributors HCG was involved in study design, screened all studies, summarised and interpreted studies and drafted the manuscript. RTW and DMA were involved in study design, interpretation of studies, reviewed and edited versions of the manuscript. NK was involved in study design and reviewed and commented on versions of the manuscript.

Funding The authors gratefully acknowledge the funding received from NIHR Greater Manchester Primary Care Patient Safety Translational Research Centre. This publication is independent research by the NIHR Greater Manchester Primary Care Patient Safety Translational Research Centre. The views expressed are those of the authors and not necessarily those of the NHS, the NIHR or the Department of Health.

Competing interests None declared.

Provenance and peer review Not commissioned; externally peer reviewed.

Data sharing statement No additional data are available.

Open Access This is an Open Access article distributed in accordance with the terms of the Creative Commons Attribution (CC BY 4.0) license, which permits others to distribute, remix, adapt and build upon this work, for commercial use, provided the original work is properly cited. See: http:// creativecommons.org/licenses/by/4.0/

\section{REFERENCES}

1. World Health Organization. Preventing suicide A global imperative [Internet]. 2014 (cited 12 September 2014). http://www.who.int/ mental_health/suicide-prevention/en/

2. World Health Organization. Mental Health Action Plan 2013-2020 [Internet]. 2013 (cited 7 August 2014). http://www.who.int/mental_ health/publications/action_plan/en/

3. Christiansen E, Jensen BF. Risk of repetition of suicide attempt, suicide or all deaths after an episode of attempted suicide: a register-based survival analysis. Aust $N Z J$ Psychiatry 2007:41:257-65

4. HM Government. Preventing suicide in England: A cross-government outcomes strategy to save lives [Internet]. 2012 (cited 7 August 2014). https://www.gov.uk/government/publications/suicideprevention-strategy-launched

5. Hawton K, Van Heeringen K. Suicide. Lancet 2009;373:1372-81.

6. Harris EC, Barraclough BM. Suicide as an outcome for mental disorders. A meta-analysis. Br J Psychiatry 1997;170:205-28.

7. Singhal A, Ross J, Seminog O, et al. Risk of self-harm and suicide in people with specific psychiatric and physical disorders: comparisons between disorders using English national record linkage. J R Soc Med 2014;107:194-204.

8. Qin P, Webb R, Kapur N, et al. Hospitalization for physical illness and risk of subsequent suicide: a population study. J Intern Med 2014:273:48-58.

9. Webb RT, Kontopantelis E, Doran T, et al. Suicide risk in primary care patients with major physical diseases. Arch Gen Psychiatry 2012;69:256-64.

10. Lavigne JE, Au A, Jiang R, et al. Utilization of prescription drugs with warnings of suicidal thoughts and behaviours in the USA and the US Department of Veterans Affairs, 2009. J Pharm Health Serv Res 2012;3:157-63.

11. Posner K, Oquendo MA, Gould M, et al. Columbia Classification Algorithm of Suicide Assessment (C-CASA): classification of suicidal events in the FDA's pediatric suicidal risk analysis of antidepressants. Am J Psychiatry 2007;164:1035-43.

12. Gibbons RD, Mann JJ. Strategies for quantifying the relationship between medications and suicidal behaviour: what has been learned? Drug Saf 2011;34:375-95.

13. Reith DM, Edmonds L. Assessing the role of drugs in suicidal ideation and suicidality. CNS Drugs 2007;21:463-72.

14. Jepsen P, Johnsen SP, Sorensen HT. Risk of Suicide in Users of Cardiovascular Drugs. Am J Cardiovasc Drugs 2003;3:163-7.

15. Ferrer P, Ballarin E, Sabate M, et al. Antiepileptic drugs and suicide: a systematic review of adverse effects. Neuroepidemiol 2014:42:107-20.

16. Gelder MG, Mayou R, Cowen P. Shorter Oxford textbook of psychiatry. 4th edn. Oxford: Oxford University Press, 2001.

17. Association AP. Diagnostic and statistical manual of mental disorders. 5th edn. Arlington: American Psychiatric Publishing, 2013.

18. Joint Formulary Committee. British national formulary. 68th edn. London: BMJ Group and Pharmaceutical Press, 2014

19. Teicher M, Glod C, Cole J. Emergence of intense suicidal preoccupation during fluoxetine treatment. $A m \mathrm{~J}$ Psychiatry 1990;147:207-10.

20. Moher D, Liberati A, Tetzlaff J, et al. Preferred Reporting Items for Systematic Reviews and Meta-Analyses: the PRISMA statement. PLoS Med 2009;6:e1000097.

21. Neyarapally GA, Hammad TA, Pinheiro SP, et al. Review of quality assessment tools for the evaluation of pharmacoepidemiological safety studies. BMJ Open 2012;2:pii: e001362.

22. Arana A, Wentworth CE, Ayuso-Mateos JL, et al. Suicide-related events in patients treated with antiepileptic drugs. $N$ Engl $J$ Med 2010;363:542-51.

23. Gibbons RD, Hur K, Brown $\mathrm{CH}$, et al. Gabapentin and suicide attempts. Pharmacoepidemiol Drug Saf 2010;19:1241-7.

24. Nilsson L, Ahlbom A, Farahmand BY, et al. Risk factors for suicide in epilepsy: a case control study. Epilepsia 2002;43:644-51.

25. Olesen JB, Hansen PR, Erdal J, et al. Antiepileptic drugs and risk of suicide: a nationwide study. Pharmacoepidemiol Drug Saf 2010;19:518-24.

26. Patorno E, Bohn RL, Wahl PM, et al. Anticonvulsant medications and the risk of suicide, attempted suicide, or violent death. JAMA 2010;303:1401-9. 
27. Callréus T, Agerskov Andersen U, Hallas J, et al. Cardiovascular drugs and the risk of suicide: a nested case-control study. Eur J Clin Pharmacol 2007;63:591-6.

28. Gasse C, Derby LE, Vasilakis C, et al. Risk of suicide among users of calcium channel blockers: population based, nested case control study. BMJ 2000;320:1251.

29. Haukka J, Niskanen L, Partonen T, et al. Statin usage and all-cause and disease-specific mortality in a nationwide study. Pharmacoepidemiol Drug Saf 2012;21:61-9.

30. Lindberg G, Bingefors K, Ranstam J, et al. Use of calcium channe blockers and risk of suicide: ecological findings confirmed in population based cohort study. BMJ 1998;316:741-5.

31. Sørensen HT, Mellemkjaer L, Olsen JH. Risk of suicide in users of beta-adrenoceptor blockers, calcium channel blockers and angiotensin converting enzyme inhibitors. Brit J Clin Pharmacol 2001;52:313-18

32. Jick H, Hagberg KW, Egger P. Rate of suicide in patients taking montelukast. Pharmacotherapy 2009;29:165-6.

33. Schumock GT, Stayner LT, Valuck RJ, et al. Risk of suicide attempt in asthmatic children and young adults prescribed leukotriene-modifying agents: a nested case-control study. J Allergy Clin Immunol 2012;130:368-75

34. Jick SS, Kremers HM, Vasilakis-Scaramozza C. Isotretinion use and risk of depression, psychotic symptoms, suicide, and attempted suicide. Arch Dermatol 2000;136:1231-6.

35. Sundström A, Alfredsson L, Sjölin-Forsberg G, et al. Association of suicide attempts with acne and treatment with isotretinoin: retrospective Swedish cohort study. BMJ 2010;341:c8512.

36. Fardet L, Petersen I, Nazareth I. Suicidal behavior and severe neuropsychiatric disorders following glucocorticoid therapy in primary care. Am J Psychiatry 2012;169:491-7.

37. Fardet L, Nazareth I, Whitaker HJ, et al. Severe neuropsychiatric outcomes following discontinuation of long-term glucocorticoid therapy: a cohort study. J Clin Psychiatry 2013;74:e281-6.

38. Jick SS, Vasilakis C, Martinez C, et al. A study of the relation of exposure to quinolones and suicidal behaviour. Brit J Clin Pharmacol 1998;45:77-81.

39. Gibbons RD, Mann JJ. Varenicline, smoking cessation, and neuropsychiatric adverse events. Am J Psychiatry 2013;170:1460-7.

40. Voaklander DC, Rowe BH, Dryden DM, et al. Medical illness, medication use and suicide in seniors: a population-based case-control study. J Epidemiol Community Health 2008;62:138-46.

41. Bergman U, Isacsson G. Use of calcium channel blockers and risk of suicide: Independent studies are needed before causality is established. BMJ 1998;317:1076.

42. Chen YT, Makuch RW. Use of calcium channel blockers and risk of suicide. Perscriptions for particular drug are influenced by numerous factors. BMJ 1998;317:1077.

43. Antiepileptic drugs and suicidality. US Department of Health and Human Services Food and Drug Administration [Internet]. Silver Spring: FDA. 2008 (cited 26 June 2014]. http://www.fda.gov/ohrms/ dockets/ac/08/briefing
44. Mula M, Kanner AM, Schmitz B, et al. Antiepileptic drugs and suicidality: an expert consensus statement from the Task Force on Therapeutic Strategies of the ILAE Commission on Neuropsychobiology. Epilepsia 2013;54:199-203.

45. Hesdorffer DC, Kanner AM. The FDA alert on suicidality and antiepileptic drugs: Fire or false alarm? Epilepsia 2009;50:978-86.

46. Gunnell D, Irvine D, Wise L, et al. Varenicline and suicidal behaviour: a cohort study based on data from the General Practice Research Database. BMJ 2009;339:b3805.

47. Thomas $\mathrm{KH}$, Martin RM, Davies NM, et al. Smoking cessation treatment and risk of depression, suicide, and self harm in the Clinical Practice Research Datalink: prospective cohort study. BMJ 2013;347: 55704

48. FDA Presentations for the October 16, 2014 Joint Meeting of the Psychopharmacologic Drugs Advisory Committee and the Drug Safety and Risk Management Advisory Committee: US Department of Health and Human Services Food and Drug Administration [Internet]. Silver Spring: FDA. 2014 (cited 26 January 2015). http:// www.fda.gov/AdvisoryCommittees/CommitteesMeetingMaterials/. Drugs/PsychopharmacologicDrugsAdvisoryCommittee/ucm420257. htm

49. Medicines and Healthcare Regulatory Authority. Review of isotretinoin and psychiatric adverse reactions. London: MHRA, 2014.

50. Thomas $\mathrm{KH}$, Martin RM, Potokar J, et al. Reporting of drug induced depression and fatal and non-fatal suicidal behaviour in the UK from 1998 to 2011. BMC Pharmacol Toxicol 2014;15:54

51. Christensen J, Vestergaard M, Mortensen PB, et al. Epilepsy and risk of suicide: a population-based case-control study. Lancet Neurol 2007;6:693-8

52. Webb R, Lichtenstein $\mathrm{P}$, Larsson $\mathrm{H}$, et al. Suicide, hospital-presenting suicide attempts and criminality in bipolar disorder: examination of risk for multiple adverse outcomes. J Clin Psychiatry 2014;75: e809-e16.

53. Hill AB. The environment and disease: association Or Causation? Proc R Soc Med 1965;58:295-300.

54. Piromohamed $M$, Park $B$. Adverse drug reactions: back to the future. Br J Clin Pharmacol 2003;55:486-92.

55. Kalinin VV. Suicidality and antiepileptic drugs: is there a link? Drug Saf 2007;30:123-42.

56. Mann JJ. Neurobiology of suicidal behaviour. Nat Rev Neurosci 2003;4:819-28

57. Yang CC, Jick SS, Jick $\mathrm{H}$. Lipid-lowering drugs and the risk of depression and suicidal behavior. Arch Intern Med 2003;163:1926-32.

58. Kapur N. Non-suicidal self-injury v. attempted suicide: new diagnosis or false dichotomy? Br J Psychiatry 2013;202:326-8.

59. Retterstol N. Suicide: a European perspective. Cambridge: Cambridge University Press, 1993:253.

60. Linsley KR, Schapira K, Kelly TP. Open verdict v. suicideimportance to research. Br J Pscyhiatry 2001;178:465-8.

61. Suissa S. Immortal time bias in pharmaco-epidemiology. Am J Epidemiol 2008;167:492-9. 